\title{
Natural history of coexistent tricuspid regurgitation in patients with degenerative mitral valve disease: Implications for future guidelines
}

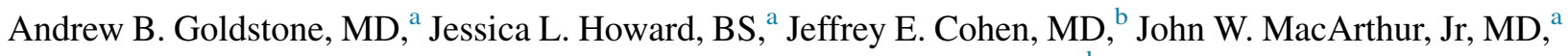 \\ Pavan Atluri, MD, ${ }^{\mathrm{a}}$ James N. Kirkpatrick, MD, ${ }^{\mathrm{c}}$ and Y. Joseph Woo, $\mathrm{MD}^{\mathrm{b}}$
}

\begin{abstract}
Objective: The management of coexistent tricuspid regurgitation in patients with mitral regurgitation remains controversial. We sought to define the incidence and natural history of coexistent tricuspid regurgitation in patients undergoing isolated mitral surgery for degenerative mitral regurgitation, as well as the effect of late secondary tricuspid regurgitation on cardiovascular symptom burden and survival.
\end{abstract}

\begin{abstract}
Methods: To minimize confounding, analysis was limited to 495 consecutive patients who underwent isolated mitral surgery for degenerative mitral valve disease between 2002 and 2011. Patients with coexistent severe tricuspid regurgitation were excluded because such patients typically undergo concomitant tricuspid intervention.

Results: Grade 1 to 3 coexistent tricuspid regurgitation was present in 215 patients (43\%) preoperatively. Actuarial freedom from grade 3 to 4 tricuspid regurgitation 1, 5, and 9 years after surgery was $100 \% \pm 0 \%$, $90 \% \pm 2 \%$, and $64 \% \pm 7 \%$, respectively. Older age $(P<.001)$ and grade of preoperative tricuspid regurgitation $(P=.006)$ independently predicted postoperative progression of tricuspid regurgitation on multivariable analysis. However, when limited to patients with mild or absent tricuspid regurgitation, indexed tricuspid annular diameter was the only significant risk factor for late tricuspid regurgitation $(P=.04)$. New York Heart Association functional class and long-term survival did not worsen with development of late secondary tricuspid regurgitation $(P=.4$ and $P=.6$, respectively). However, right ventricular dysfunction was significantly more common in patients with more severe late tricuspid regurgitation $(P=.007)$.
\end{abstract}

Conclusions: Despite durable correction of degenerative mitral regurgitation, less than severe tricuspid regurgitation is likely to progress after surgery if uncorrected. Given the low incremental risk of tricuspid annuloplasty, a more aggressive strategy of concomitant tricuspid repair may be warranted. (J Thorac Cardiovasc Surg 2014;148:2802-10)

See related commentary on pages $2810-2$.

The contemporary management of coexistent tricuspid regurgitation (TR) in patients with mitral regurgitation (MR) remains controversial. As such, the frequency of tricuspid valve repair varies significantly. ${ }^{1-3}$ Early data that surgical correction of mitral valve disease secondarily alleviates functional TR by improving left heart hemodynamics, ventricular geometry, and tricuspid valve

\footnotetext{
From the Division of Cardiovascular Surgery, ${ }^{\mathrm{a}}$ Department of Surgery, Perelman School of Medicine, University of Pennsylvania, Philadelphia, Pa; Department of Cardiothoracic Surgery, ${ }^{\mathrm{b}}$ Stanford University School of Medicine, Stanford, Calif; and the Division of Cardiology, ${ }^{\mathrm{c}}$ Department of Medicine, Perelman School of Medicine, University of Pennsylvania, Philadelphia, Pa.

Disclosures: Authors have nothing to disclose with regard to commercial support.

Read at the 94th Annual Meeting of The American Association for Thoracic Surgery, Toronto, Ontario, Canada, April 26-30, 2014.

Received for publication April 28, 2014; revisions received July 27, 2014; accepted for publication Aug 1, 2014; available ahead of print Sept 11, 2014.

Address for reprints: Y. Joseph Woo, MD, Department of Cardiothoracic Surgery, Stanford University, Falk Bldg, CV-235, 300 Pasteur Dr, Stanford, CA 94305-5407 (E-mail: joswoo@stanford.edu).

$0022-5223 / \$ 36.00$

Copyright (C) 2014 by The American Association for Thoracic Surgery

http://dx.doi.org/10.1016/j.jtcvs.2014.08.001
}

strain have led many physicians to manage coexistent TR conservatively. ${ }^{2,45}$ However advocates for a more aggressive surgical strategy maintain that the development of late significant TR after isolated mitral valve surgery is not uncommon, ${ }^{6-8}$ and untreated TR is independently associated with significantly worse cardiovascular symptom burden and survival. ${ }^{9}$ Furthermore, late surgical intervention for recurrent TR is often prohibitively dangerous, ${ }^{10,11}$ whereas concomitant tricuspid annuloplasty presents lower risk and durably prevents recurrent TR. ${ }^{12-15}$ As such, a prophylactic strategy of routine concomitant tricuspid valve repair has recently been proposed.

Although generally absent from society guidelines, disease etiology is paramount in managing MR and may significantly influence progression of functional TR. Because increasing evidence supports early surgical repair of patients with severe degenerative MR before the development of adverse sequelae of longstanding disease ${ }^{16}$ (eg, ventricular remodeling and dysfunction, pulmonary hypertension, and atrial fibrillation), perhaps this patient population is less likely to develop late significant TR if left untreated. Furthermore, guidelines ${ }^{17,18}$ have become increasingly aggressive with regard to concomitant tricuspid valve repair despite a large report of patients with degenerative MR demonstrating that 


\section{Abbreviations and Acronyms \\ MR = mitral regurgitation \\ NYHA $=$ New York Heart Association \\ $\mathrm{TR}=$ tricuspid regurgitation}

progression of nonsevere TR is unlikely in this population. ${ }^{2}$ Clearly, more data are needed to better inform the clinical decision making surrounding coexistent TR. Thus, we sought to define the incidence and natural history of coexistent TR in patients undergoing isolated mitral valve surgery for degenerative mitral valve disease, as well as determine the influence of late secondary TR on cardiovascular symptom burden and survival.

\section{PATIENTS AND METHODS}

Between 2002 and 2011, 4241 patients underwent mitral valve surgery at our institution. Isolated mitral valve surgery, defined as any mitral valve procedure performed in the absence of a major concomitant procedure (eg, coronary bypass, other valve surgery, or aortic surgery) was performed in 1011 patients. To minimize confounding, analysis was limited to 495 consecutive patients who underwent isolated mitral valve surgery for chronic severe MR secondary to mitral valve prolapse. Patients with preoperative coexistent severe TR were excluded. Also excluded were patients with primary pulmonary disease or structural tricuspid valve abnormalities. Data were in part retrieved from the University of Pennsylvania's prospective Society of Thoracic Surgeons registry and partially obtained from each patient's medical record. These data were approved for use in research by the University of Pennsylvania Institutional Review Board with a waiver of patient consent.

All patients underwent mitral valve surgery for severe MR due to leaflet prolapse. The techniques used during mitral valve surgery have been previously described. ${ }^{19-21}$ Posterior leaflet prolapse was typically corrected with triangular or quadrangular resection of the prolapsed segment, or nonresectional leaflet remodeling. Anterior leaflet prolapse was characteristically repaired with polytetrafluoroethylene neochords. All repairs were supplemented with a circumferential or partial ring annuloplasty sized to the surface area of the anterior leaflet.

For each patient, pre- and postoperative TR was graded on a standard, semiquantitative 0 to 4 scale: grade $0=$ none, grade $1=$ trivial, grade $2=$ mild, grade $3=$ moderate, and grade $4=$ severe. Postdischarge transthoracic echocardiograms were available for 362 patients $(73 \%)$, with a median follow-up of 3.4 years (minimum 18 days, maximum 11.1 years). Postdischarge echocardiograms were interpreted by the referring cardiologist. Clinical follow-up data were collected from postoperative visit data and records from referring cardiologists (median follow-up was 4.5 years with an interquartile range of 3.2-6.3 years).

A subgroup analysis of 127 patients $(25 \%)$ was conducted to elucidate the prognostic value of tricuspid annular dilation. Using intraoperative transesophageal echocardiograms, the tricuspid valve annulus was measured from the midesophageal 4-chamber and short axis views before mitral valve intervention. Measurements were obtained at end diastole and end systole by a single, level-III trained echocardiographer.

Continuous variables are expressed as the mean \pm standard deviation and categorical variables are presented as proportions. Comorbid diagnoses and perioperative outcome variables adhere to the definitions of the Society of Thoracic Surgeons. Changes in coexistent TR from preoperative values to pre- and postbypass values were compared using the Wilcoxon signed-rank test. Differences in tricuspid annular diameter were compared with 1-way analysis of variance tests. Cox regression models were used to identify predictors of TR progression during follow-up. The multivariable model considered all variables found to be significant on univariate analysis $(P<.05)$ in a stepwise fashion (forward and backward selection yielded equivalent models). Survival and cardiovascular symptom burden analyses were performed using Kaplan-Meier analysis with log-rank tests. All tests were 2-tailed. The statistical analysis was performed using IBM-SPSS Statistics for Macintosh, version 20.0 (IBM-SPSS, Inc, Armonk, NY)

\section{RESULTS}

A total of 495 patients who underwent isolated mitral valve surgery met the inclusion criteria for the study. All patients underwent mitral valve repair. Baseline demographics and comorbidity profiles are summarized in Table 1. Preoperatively, nearly one-third of patients were asymptomatic based on New York Heart Association (NYHA) functional class assessment $(\mathrm{n}=116 ; 32.3 \%)$ and 56 patients $(11.3 \%)$ had a history of atrial fibrillation or flutter. The majority of patients had no evidence of coexistent TR on preoperative echocardiography $(n=280$; $56.6 \%)$. Although 146 patients $(29.5 \%)$ had grade 2 coexistent TR or higher on preoperative echocardiography, none was deemed by the attending surgeon to require concomitant intervention at the time of mitral valve surgery.

\section{Changes in Coexistent TR}

We compared TR grade at preoperative and intraoperative time points in the 39 patients with grade 3 preoperative TR (Figure 1). Intraoperative grade of TR (pre- and postcardiopulmonary bypass) was significantly lower than preoperative TR grade. In fact, only 1 patient was found to have grade 3 TR on intraoperative echocardiographic assessment. Grade of TR did not significantly differ between pre- and postcardiopulmonary bypass time points.

Applying Kaplan-Meier analysis to the overall cohort revealed actuarial freedom from grade 3 or greater TR at 1,5 , and 9 years after surgery was $100 \% \pm 1 \%$, $90 \% \pm 2 \%$, and $64 \% \pm 7 \%$, respectively. When stratified by preoperative TR grade, patients with greater degrees of coexistent TR demonstrated faster progression to significant postoperative TR $(P<.001)$ (Figure 2). During the same time interval, freedom from recurrent grade 3 or greater MR at 1,5 , and 9 years after surgery was $99 \% \pm 1 \%, 92 \% \pm 2 \%$, and $82 \% \pm 5 \%$, respectively. One patient developed grade 4 MR during the study period and 16 patients developed grade $3 \mathrm{MR}$, but severity of recurrent MR was not found to be significantly associated with progression of TR in univariate or multivariable analyses. Out of 22 fixed-effect preoperative variables examined in univariate analyses, 8 were found to be significant predictors of TR progression during follow-up. However only advanced age and preoperative TR grade remained significant risk factors for progression of TR on multivariable analysis (Table 2). It should be noted that although preoperative atrial fibrillation failed to remain significant in multivariable analysis, postoperative atrial 
TABLE 1. Demographics and risk profile of study population $(\mathrm{N}=495)$

\begin{tabular}{lc}
\multicolumn{1}{c}{ Variable } & Value \\
\hline Demographic & \\
Age (y) & $57.5 \pm 12.5$ \\
Female gender & $174(38.2)$ \\
Comorbidities & \\
Atrial fibrillation & $56(11.3)$ \\
Renal failure & $7(1.4)$ \\
Hypertension & $211(42.6)$ \\
Chronic lung disease & $41(8.3)$ \\
Peripheral arterial disease & $6(1.2)$ \\
Cerebrovascular disease & $15(3.0)$ \\
Previous cardiac surgery & $30(6.1)$ \\
New York Heart Association functional class & \\
I & $160(32.3)$ \\
II & $262(52.9)$ \\
III & $68(13.7)$ \\
IV & $5(1.0)$ \\
Mean pulmonary artery pressure $(\mathrm{mm} \mathrm{Hg})$ & $21.5 \pm 8.9$ \\
Ejection fraction $(\%)$ & $58.3 \pm 8.4$ \\
Tricuspid regurgitation grade & \\
0 & $280(56.6)$ \\
1 & $69(13.9)$ \\
2 & $107(21.6)$ \\
3 & $39(7.9)$ \\
Tricuspid annular diameter $(\mathrm{mm})$ & $40.1 \pm 5.6$ \\
Indexed tricuspid annular diameter $\left(\mathrm{mm} / \mathrm{m}^{2}\right)$ & $20.9 \pm 3.2$ \\
Leaflet prolapse & \\
Posterior & $371(74.9)$ \\
Anterior & $28(5.7)$ \\
Bileaflet & $96(19.4)$ \\
\hline
\end{tabular}

Values for continuous variables are presented as means \pm standard deviation. Others are presented as $\mathrm{n}(\%)$

fibrillation was present in 116 patients $(23.4 \%)$ and was both significant in univariate and multivariable analyses (hazard ratio, 2.0; $P=.03$ ) in addition to age and preoperative TR grade.

\section{Tricuspid Annular Dilation and Late TR}

Mean indexed tricuspid annular diameter increased with increasing severity of preoperative TR (grade $0,20.4 \pm 3.2$ $\mathrm{mm} / \mathrm{m}^{2}$; grade 1 and $2,20.8 \pm 3.2 \mathrm{~mm} / \mathrm{m}^{2}$; and grade $3,23.4$ $\left.\pm 2.5 \mathrm{~mm} / \mathrm{m}^{2} ; P=.005\right)$. In fact, all but 1 patient with grade 3 preoperative TR had an indexed tricuspid annular diameter greater than $21 \mathrm{~cm} / \mathrm{m}^{2}$ - the current recommended threshold for repair. Although a significant predictor of late TR on univariate analysis, indexed tricuspid annular diameter failed to remain significant on multivariable analysis (Table 2). However in patients with less than grade 3 preoperative TR, indexed tricuspid annular diameter was the only independent risk factor for progression of TR (hazard ratio, $6.3 ; P=.04$ ). Nonindexed tricuspid annular diameter was not a significant predictor of late TR in univariate or multivariable analyses.

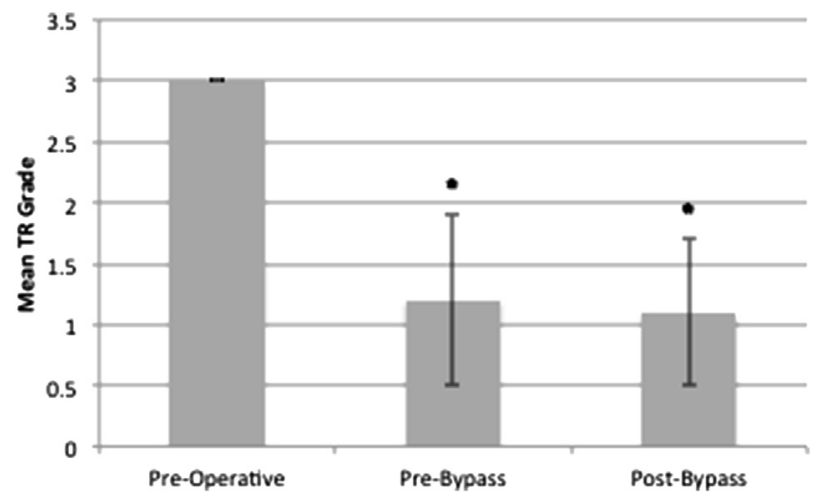

FIGURE 1. Mean grade of tricuspid regurgitation $(T R)$ significantly decreases from preoperative value with the institution of cardiopulmonary bypass in patients with grade $3 \mathrm{TR}(\mathrm{N}=39)$. $* P<.001$ compared with preoperative value.

\section{Burden of Postoperative TR}

Despite a $63 \% \pm 14 \%$ freedom from grade 3 or greater TR 10 years after mitral valve surgery, no patient underwent late intervention on the tricuspid valve. Freedom from NYHA functional class III or IV at 1, 5, and 9 years after surgical correction of MR was $99 \% \pm 1 \%, 97 \% \pm 1 \%$, and $96 \% \pm 2 \%$, respectively. Furthermore, cardiovascular symptom burden as assessed by NYHA functional class did not significantly worsen with development of greater degrees of late secondary TR $(P=.4)$. On the other hand, freedom from moderate or greater right ventricular dysfunction at 1,5 , and 9 years after surgery was $98 \% \pm$ $1 \%, 90 \% \pm 3 \%$, and $70 \% \pm 9 \%$, respectively, and right ventricular dysfunction was significantly associated with higher postoperative TR grade $(P=.007)$ (Figure 3$)$.

There were no deaths before hospital discharge. Overall survival 1,5 , and 10 years after surgery was $99 \% \pm 1 \%$, $97 \% \pm 1 \%$, and $96 \% \pm 1 \%$, respectively. Survival trends did not significantly differ when stratified by development of postoperative TR $(P=.6)$.

\section{DISCUSSION}

The management of coexistent TR in patients with chronic MR remains controversial. Although some advocate that relief of left-sided valve dysfunction will durably correct functional TR, society guidelines are becoming increasingly aggressive with regard to the implementation of concomitant tricuspid valve repair. Our results support a more aggressive approach. By analyzing clinical and echocardiographic data from a homogenous population of patients undergoing isolated mitral valve surgery for degenerative mitral valve disease, we found that postoperative TR progression is not uncommon, and is associated with worsening right ventricular function.

Coexistent TR is relatively common in patients referred for chronic severe MR. ${ }^{2,12,22}$ Although early experience 


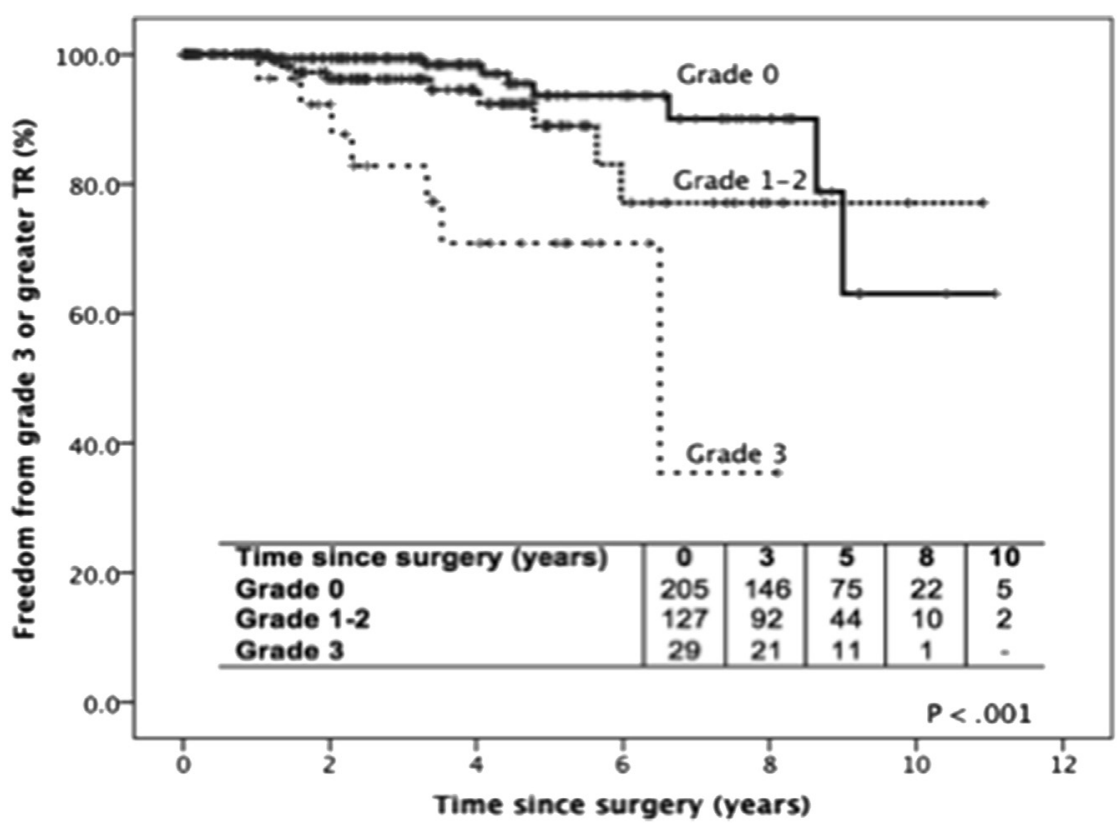

FIGURE 2. Freedom from grade 3 or greater tricuspid regurgitation $(T R)$ after isolated mitral valve surgery stratified by preoperative TR grade.

with mitral valve replacement suggested that coexistent TR is consistently improved with correction of left-sided valve dysfunction, ${ }^{4,5}$ it was later realized that in select patients TR may gradually worsen, resulting in debilitating right heart failure requiring reoperation. Unfortunately, untreated TR negatively influences functional status and long-term survival, ${ }^{9}$ and late surgical intervention for recurrent TR is often prohibitively dangerous. ${ }^{10,23,24}$ Recent studies have demonstrated both an improvement in NYHA functional class ${ }^{8}$ as well as a survival benefit ${ }^{25}$ in patients who undergo concomitant tricuspid annuloplasty at the time of mitral valve surgery compared with those who do not. As such, there has been increasing support for a more liberal application of tricuspid valve repair in patients with coexistent TR who present for mitral valve surgery. However the question remains, who should undergo concomitant tricuspid repair?

The American College of Cardiology and American Heart Association have taken a more aggressive approach in the most recent update on the management of valvular heart disease. Current guidelines now advise concomitant tricuspid valve repair at the time of mitral valve surgery if coexistent TR is severe (class I), and suggest repair if TR is less than severe in patients with tricuspid annular dilation or pulmonary hypertension (class IIa). ${ }^{17}$ The 2012 iteration of European guidelines is similar to that of the United States, assigning a class IIa recommendation to tricuspid repair in patients undergoing any left-sided valve surgery with mild or moderate coexistent TR if the annulus is $>40$ $\mathrm{mm}$ or $>21 \mathrm{~mm} / \mathrm{m}^{2}$ (albeit all level of evidence C). ${ }^{18}$ Yet, absent from all guidelines is an emphasis on disease etiology. A uniform approach to functional TR assumes that TR progresses similarly after correction of all types of left-sided valve dysfunction.

Etiologies of mitral valve disease are quite distinct and convey disparate prognoses. For example, the left ventricular dysfunction observed in ischemic and nonischemic cardiomyopathy may commonly compromise right ventricular function, leading to significant tricuspid annular dilation and TR. Repair of ischemic MR is also significantly less effective than that of MR due to leaflet prolapse. Similarly, mitral valve repair of rheumatic valve disease does not halt the continued valvulopathic processes that underlie the disease. In such patients, the development of late recurrent TR is quite common. In fact, in the setting of ischemic or rheumatic mitral valve disease, clinically significant late TR has been shown to develop in $37 \%$ to $49 \%$ of patients. ${ }^{6,7}$ Even more striking, Matsunaga and Duran ${ }^{7}$ documented rapid progression to greater-than-moderate TR in $74 \%$ of patients just 3 years after surgery.

To date few studies have examined the natural history and clinical influence of coexistent TR in those with the most frequent mitral valve condition encountered in developed nations-degenerative mitral valve disease., ${ }^{2,12}$ In an analysis of 696 patients with mitral valve prolapse and clinically silent functional TR undergoing isolated mitral valve repair, Yilmaz and colleagues ${ }^{2}$ found that clinically important TR progression is unlikely to occur after mitral valve repair. Thus, they argue that concomitant surgical tricuspid intervention for functional TR is rarely necessary in patients undergoing isolated mitral valve repair for leaflet 
TABLE 2. Risk factors for development of grade 3 to 4 tricuspid regurgitation

\begin{tabular}{|c|c|c|c|c|}
\hline \multirow[b]{2}{*}{ Variable } & \multicolumn{2}{|c|}{ Univariate } & \multicolumn{2}{|c|}{ Multivariable } \\
\hline & $\begin{array}{c}\text { Hazard } \\
\text { ratio }\end{array}$ & $\begin{array}{c}P \\
\text { value }\end{array}$ & $\begin{array}{c}\text { Hazard } \\
\text { ratio }\end{array}$ & $\begin{array}{c}P \\
\text { value }\end{array}$ \\
\hline \multicolumn{5}{|l|}{ Demographic } \\
\hline Age & 1.1 & $<.001$ & 1.1 & $<.001$ \\
\hline Gender (female:male) & 1.8 & .048 & & \\
\hline Body mass index & 0.9 & .099 & & \\
\hline \multicolumn{5}{|l|}{ Comorbidities } \\
\hline Previous atrial fibrillation & 2.3 & .049 & & \\
\hline Pulmonary hypertension* & 0.3 & .088 & & \\
\hline Renal failure & 2.3 & .415 & & \\
\hline Hypertension & 1.3 & .408 & & \\
\hline Diabetes & 1.0 & .989 & & \\
\hline Dyslipidemia & 1.1 & .697 & & \\
\hline Chronic lung disease & 1.8 & .335 & & \\
\hline Peripheral arterial disease & 4.8 & .031 & & \\
\hline Cerebrovascular disease & 0.9 & .952 & & \\
\hline Previous cardiac intervention & 3.2 & .005 & & \\
\hline $\begin{array}{l}\text { Recent congestive heart failure } \\
\text { exacerbation }\end{array}$ & 1.9 & .042 & & \\
\hline $\begin{array}{l}\text { New York Heart Association } \\
\text { functional class (vs class I) }\end{array}$ & & .016 & & \\
\hline II & 1.4 & & & \\
\hline III & 1.8 & & & \\
\hline IV & 28.6 & & & \\
\hline Mean pulmonary artery pressure & 1.0 & .779 & & \\
\hline Ejection fraction & 1.0 & .324 & & \\
\hline $\begin{array}{l}\text { Tricuspid regurgitation grade } \\
\text { (vs grade } 0 \text { ) }\end{array}$ & & .001 & & .006 \\
\hline 1 & 1.3 & & 1.4 & \\
\hline 2 & 3.4 & & 3.4 & \\
\hline 3 & 4.5 & & 3.1 & \\
\hline Tricuspid annular diameter & 1.0 & .964 & & \\
\hline Indexed tricuspid annular diameter & 6.1 & .020 & & \\
\hline Leaflet prolapse (vs posterior) & & .213 & & \\
\hline Anterior & 1.2 & & & \\
\hline Bileaflet & 2.1 & & & \\
\hline Right minithoracotomy approach & 0.9 & .667 & & \\
\hline
\end{tabular}

prolapse. Although overall TR grade increased only slightly 5 years after surgery, the mean TR grade remained only mild. Yet, an average may not be the ideal method for analyzing ordinal data, particularly for a semiquantitative variable like TR grade. When presented as a proportion, nearly $30 \%$ of patients exhibited grade 3 or greater TR after 5 years of follow-up. ${ }^{2}$ However, recurrent TR did not negatively influence survival and only 1 patient required reoperation for severe symptomatic TR.

We also examined a homogenous population of patients undergoing isolated mitral valve surgery for degenerative MR. Recurrent TR was not uncommon-36\% of patients were estimated to have moderate or greater TR 9 years after surgery. Nevertheless, development of significant TR during follow-up did not influence survival or NYHA functional class. Because patients with degenerative mitral valve disease are increasingly referred for surgery earlier in the disease process, it is not surprising that overall survival was excellent. Accordingly a tremendous number of patients would be necessary to power a study to detect significant differences in survival. Furthermore, NYHA functional class is not an ideal metric for symptoms due to right heart failure from untreated TR. Although no patient underwent reoperation for significant TR, patients with higher postoperative TR grades more frequently had significant right ventricular dysfunction during the follow-up period. Desai and colleagues ${ }^{12}$ demonstrated the bidirectional interrelatedness of TR and right ventricular function: not only does right ventricular dysfunction affect severity of TR, but also reduced TR is associated with improved right ventricular function. Tricuspid valve repair remains the most effective treatment to improve right ventricular function. ${ }^{26}$ Thus, right ventricular function may be a better metric than NYHA functional class in this population. In fact, European guidelines now advise physicians to consider tricuspid valve repair in asymptomatic patients with worsening right ventricular function. ${ }^{18}$

Only 2 preoperative variables were identified as independent risk factors for TR progression in the overall cohort: increasing age and preoperative TR grade. In addition to those risk factors, prior investigations have documented female gender, ${ }^{2,6}$ atrial fibrillation, ${ }^{2,27}$ enlarged left atrium, ${ }^{27}$ pulmonary hypertension, ${ }^{28}$ tricuspid annular dilation, ${ }^{8,29,30}$ and diabetes mellitus ${ }^{2}$ to be independently associated with the development of late TR after left-sided valve surgery. Although a preoperative history of atrial arrhythmias was a significant univariate predictor of TR progression, in our study it failed to prove significant in the multivariable model. On the other hand, postoperative atrial fibrillation was a significant risk factor for TR progression in multivariable analysis, and thus in such patients all efforts should be made to try and maintain sinus rhythm after surgery. Interstudy differences in the risk factors identified may be a product of patient selection, because many studies have included aortic valve surgery and/or mixed etiologies of mitral valve disease. However, it may also allude to the multifactorial nature of functional $\mathrm{TR}^{28}$

It should also be noted that intraoperative echocardiographic assessment of TR does not correlate with preoperative TR grade and is not indicative of the risk of developing late TR. Afterload reduction from general anesthesia as well as volume unloading before surgery typically reduce the severity of TR noted in the operating theater. As such, surgical decision making should largely be based on preoperative imaging. In fact, preoperative TR grade strongly predicted development of late TR in a 


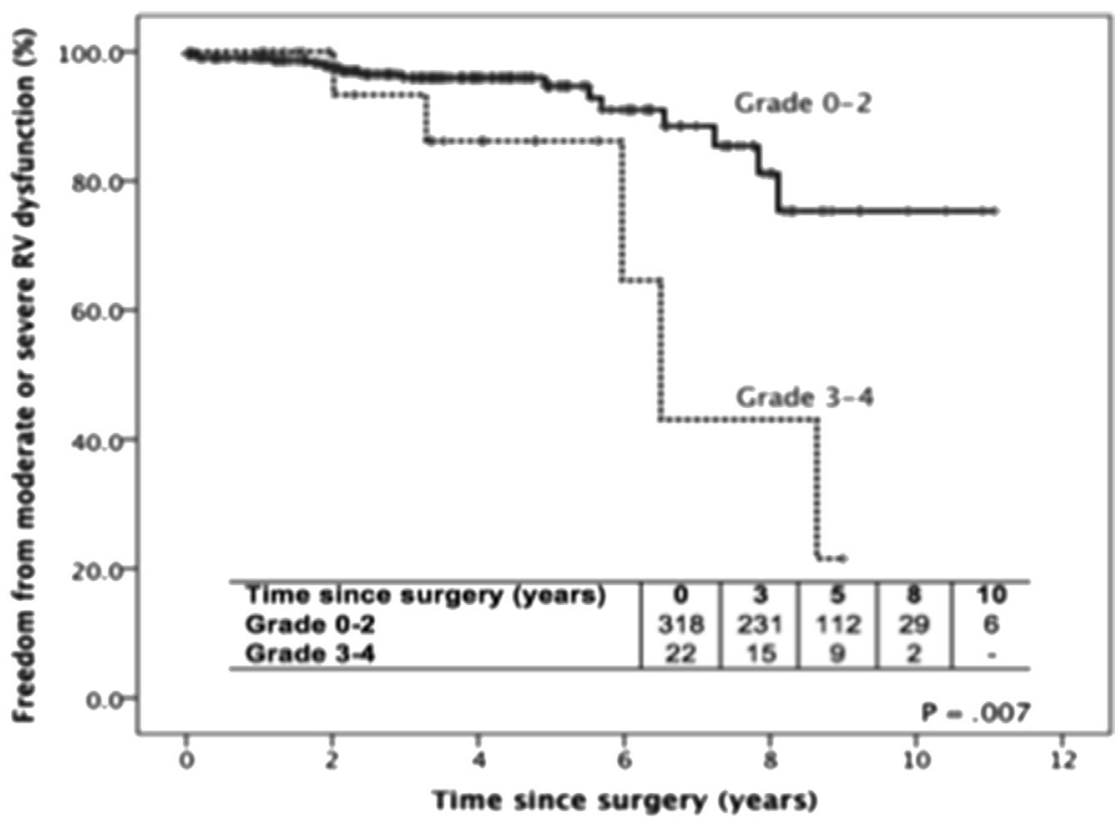

FIGURE 3. Freedom from developing moderate or severe right ventricular $(R V)$ dysfunction after isolated mitral valve surgery stratified by development of significant postoperative tricuspid regurgitation.

stepwise fashion, and more consideration should be given toward intervening on nonsevere grades of TR.

In our study population, tricuspid annular dimensions were larger in patients with higher grades of preoperative TR. In fact, all but 1 patient with grade 3 TR preoperatively had a tricuspid annular diameter above the recommended threshold for intervention, and it was this population that had the greatest risk for developing late TR. Thus, once a valve has been geometrically altered enough to permit moderate grades of TR, it is unlikely that the valve will structurally remodel enough after mitral valve repair to prevent progressive or recurrent TR. Although preoperative grade of TR was a stronger predictor of developing late TR in the overall study cohort, when the analysis was limited to patients with less than grade $3 \mathrm{TR}$, indexed tricuspid annular diameter remained the only significant risk factor for developing significant postoperative TR. Thus, it may be advised to intervene on the tricuspid valve in the setting of mild or absent TR when the indexed tricuspid annular diameter is enlarged. Furthermore, it is important to note that nonindexed tricuspid annular diameter was not a significant risk factor for progression of TR on univariate or multivariable analysis. This lack of predictive power may be due to the fact that nonindexed parameters do not account for relatively dilated annuli in smaller people.

Given that uncorrected chronic MR often results in progressive left ventricular dysfunction, pulmonary hypertension, and atrial fibrillation (all common risk factors for late TR), it was of interest to elucidate if asymptomatic individuals are less likely to develop recurrent TR. Although significant on univariate analysis, preoperative NYHA functional class failed to predict progressive postoperative TR on multivariable analysis. Thus, preoperative symptom status may be a confounder for more important variables-already having evidence of TR seems to be a much stronger predictor of whether patients are likely to develop recurrent TR after left-sided valve surgery.

We believe that our results are in accord with the updated American and European guidelines, in that patients with only mild or moderate coexistent TR (and even those without any evidence of preoperative TR) are at increased risk of developing late functional TR, especially in the setting of tricuspid annular enlargement. Although rates of TR progression appear to be less severe in patients with degenerative mitral valve disease than other etiologies, the risk of developing TR with concomitant right ventricular dysfunction is significant. Given the low incremental risk of concomitant tricuspid annuloplasty, a more liberal strategy of tricuspid valve repair may prove beneficialwherein all patients with moderate TR undergo tricuspid valve repair, and patients with mild or absent TR may undergo concomitant preventative annuloplasty when there is enlargement of the indexed tricuspid annular diameter.

\section{Limitations}

The limitations of our study include its retrospective methodology and associated biases. Of course, treatment allocation bias exists in that the decision to intervene on the tricuspid valve at the time of mitral valve surgery was at the discretion of the surgeon. Comparing our study cohort 
with similar patients who underwent concomitant tricuspid intervention would be valuable, but our limited experience with such patients $(\mathrm{N}=37)$ limits a feasible evaluation. Because echocardiographic follow-up was incomplete, we recognize the potential for under- or overestimation of the incidence of recurrent symptomatic severe TR. Also, because multiple postoperative echocardiograms were unavailable for each patient, we were unable to perform a mixed modeling analysis. ${ }^{31}$ However as in most studies, TR appears to progress over time, so it is possible that having access to echocardiograms at multiple time points may have hastened the observed rate of progression to grade 3 to 4 TR. Furthermore, we acknowledge that we are unable to quantify symptoms of right heart failure and have used NYHA functional class and echocardiographic assessment of right ventricular function as surrogate measures. Because the study occurred over a decade, practice patterns may have changed as well as the ability to quantify TR via echocardiography.

\section{CONCLUSIONS}

Despite durable correction of degenerative MR, lessthan-severe TR and even absent TR is likely to progress after surgery for degenerative MR if uncorrected. Although uncorrected TR does not appear to negatively influence survival in this population, it is associated with impaired right ventricular function. Given the low incremental risk of tricuspid annuloplasty, a more aggressive strategy of concomitant tricuspid repair may be warranted. However disease etiology and specific preoperative risk factors should be considered for appropriate patient selection. Clearly additional investigation is necessary to best inform clinical decision making in the management of this complex disease process, and hopefully a randomized clinical trial will be conducted in the near future.

\section{References}

1. Kilic A, Saha-Chaudhuri P, Rankin JS, Conte JV. Trends and outcomes of tricuspid valve surgery in North America: an analysis of more than 50,000 patients from the Society of Thoracic Surgeons database. Ann Thorac Surg. 2013;96:1546-52.

2. Yilmaz O, Suri RM, Dearani JA, Sundt TM III, Daly RC, Burkhart HM, et al. Functional tricuspid regurgitation at the time of mitral valve repair for degenerative leaflet prolapse: the case for a selective approach. J Thorac Cardiovasc Surg. 2011;142:608-13.

3. Teman NR, Huffman LC, Krajacic M, Pagani FD, Haft JW, Bolling SF "Prophylactic" tricuspid repair for functional tricuspid regurgitation. Ann Thorac Surg. 2014;97:1520-4.

4. Braunwald NS, Ross J Jr, Morrow AG. Conservative management of tricuspid regurgitation in patients undergoing mitral valve replacement. Circulation. 1967;35:I63-9.

5. Hannoush H, Fawzy ME, Stefadouros M, Moursi M, Chaudhary MA, Dunn B. Regression of significant tricuspid regurgitation after mitral balloon valvotomy for severe mitral stenosis. Am Heart J. 2004;148:865-70.

6. Porter A, Shapira Y, Wurzel M, Sulkes J, Vaturi M, Adler Y, et al. Tricuspid regurgitation late after mitral valve replacement: clinical and echocardiographic evaluation. J Heart Valve Dis. 1999;8:57-62.

7. Matsunaga A, Duran CM. Progression of tricuspid regurgitation after repaired functional ischemic mitral regurgitation. Circulation. 2005;112:I453-7.
8. Dreyfus GD, Corbi PJ, Chan KM, Bahrami T. Secondary tricuspid regurgitation or dilatation: which should be the criteria for surgical repair? Ann Thorac Surg. 2005;79:127-32.

9. Nath J, Foster E, Heidenreich PA. Impact of tricuspid regurgitation on long-term survival. J Am Coll Cardiol. 2004;43:405-9.

10. King RM, Schaff HV, Danielson GK, Gersh BJ, Orszulak TA, Piehler JM, et al. Surgery for tricuspid regurgitation late after mitral valve replacement. Circulation. 1984;70:I193-7.

11. Kuwaki K, Morishita K, Tsukamoto M, Abe T. Tricuspid valve surgery for functional tricuspid valve regurgitation associated with left-sided valvular disease. Eur J Cardiothorac Surg. 2001;20:577-82.

12. Desai RR, Vargas Abello LM, Klein AL, Marwick TH, Krasuski RA, Ye Y, et al. Tricuspid regurgitation and right ventricular function after mitral valve surgery with or without concomitant tricuspid valve procedure. J Thorac Cardiovasc Surg. 2013;146:1126-32.

13. McCarthy PM, Bhudia SK, Rajeswaran J, Hoercher KJ, Lytle BW, Cosgrove DM, et al. Tricuspid valve repair: durability and risk factors for failure. J Thorac Cardiovasc Surg. 2004;127:674-85.

14. Navia JL, Nowicki ER, Blackstone EH, Brozzi NA, Nento DE, Atik FA, et al. Surgical management of secondary tricuspid valve regurgitation: annulus, commissure, or leaflet procedure? J Thorac Cardiovasc Surg. 2010;139:1473-82.

15. Chan V, Burwash IG, Lam BK, Auyeung T, Tran A, Mesana TG, et al. Clinical and echocardiographic impact of functional tricuspid regurgitation repair at the time of mitral valve replacement. Ann Thorac Surg. 2009;88: 1209-15.

16. Suri RM, Vanoverschelde JL, Grigioni F, Schaff HV, Tribouilloy C, Avierinos JF, et al. Association between early surgical intervention vs watchful waiting and outcomes for mitral regurgitation due to flail mitral valve leaflets. JAMA. 2013;310:609-16.

17. Nishimura RA, Otto CM, Bonow RO, Carabello BA, Erwin JP III, Guyton RA, et al. 2014 AHA/ACC guideline for the management of patients with valvular heart disease: a report of the American College of Cardiology/American Heart Association Task Force on Practice Guidelines. Circulation. 2014;148:e1-132.

18. Vahanian A, Alfieri O, Andreotti F, Antunes MJ, Baron-Esquivias G, Baumgartner H, et al. Guidelines on the management of valvular heart disease (version 2012): the Joint Task Force on the Management of Valvular Heart Disease of the European Society of Cardiology (ESC) and the European Association for Cardio-Thoracic Surgery (EACTS). Eur J Cardiothorac Surg. 2012;42:S1-44.

19. Goldstone AB, Atluri P, Szeto WY, Trubelja A, Howard JL, MacArthur JW Jr, et al. Minimally invasive approach provides at least equivalent results for surgical correction of mitral regurgitation: a propensity-matched comparison. J Thorac Cardiovasc Surg. 2013;145:748-56.

20. Woo YJ, MacArthur JW Jr. Simplified nonresectional leaflet remodeling mitral valve repair for degenerative mitral regurgitation. J Thorac Cardiovasc Surg. 2012;143:749-53.

21. Goldstone AB, Woo YJ. Minimally invasive surgical treatment of valvular heart disease. Semin Thorac Cardiovasc Surg. 2014;26:36-43.

22. Cohn LH. Tricuspid regurgitation secondary to mitral valve disease: when and how to repair. J Card Surg. 1994;9:237-41.

23. Staab ME, Nishimura RA, Dearani JA. Isolated tricuspid valve surgery for severe tricuspid regurgitation following prior left heart valve surgery: analysis of outcome in 34 patients. J Heart Valve Dis. 1999;8:567-74.

24. Cohen SR, Sell JE, McIntosh CL, Clark RE. Tricuspid regurgitation in patients with acquired, chronic, pure mitral regurgitation. II. Nonoperative management, tricuspid valve annuloplasty, and tricuspid valve replacement. J Thorac Cardiovasc Surg. 1987;94:488-97.

25. Calafiore AM, Gallina S, Iaco AL, Contini M, Bivona A, Gagliardi M, et al. Mitral valve surgery for functional mitral regurgitation: should moderate-ormore tricuspid regurgitation be treated? A propensity score analysis. Ann Thorac Surg. 2009;87:698-703.

26. Ye Y, Desai R, Vargas Abello LM, Rajeswaran J, Klein AL, Blackstone EH, et al. Effects of right ventricular morphology and function on outcomes of patients with degenerative mitral valve disease. J Thorac Cardiovasc Surg. April 1, 2014 [Epub ahead of print].

27. Matsuyama K, Matsumoto M, Sugita T, Nishizawa J, Tokuda Y, Matsuo T Predictors of residual tricuspid regurgitation after mitral valve surgery. Ann Thorac Surg. 2003;75:1826-8.

28. Mutlak D, Aronson D, Lessick J, Reisner SA, Dabbah S, Agmon Y. Functional tricuspid regurgitation in patients with pulmonary hypertension: is 
pulmonary artery pressure the only determinant of regurgitation severity? Chest. 2009; 135:115-21.

29. Van de Veire NR, Braun J, Delgado V, Versteegh MI, Dion RA, Klautz RJ, et al. Tricuspid annuloplasty prevents right ventricular dilatation and progression of tricuspid regurgitation in patients with tricuspid annular dilatation undergoing mitral valve repair. J Thorac Cardiovasc Surg. 2011;141:1431-9.

30. Colombo T, Russo C, Ciliberto GR, Lanfranconi M, Bruschi G, Agati S, et al. Tricuspid regurgitation secondary to mitral valve disease: tricuspid annulus function as guide to tricuspid valve repair. Cardiovasc Surg. 2001; 9:369-77.

31. Blackstone EH. Breaking down barriers: helpful breakthrough statistical methods you need to understand better. J Thorac Cardiovasc Surg. 2001;122:430-9.

\section{Discussion}

Dr Kenton J. Zehr (Baltimore, Md). I thank the Association for the opportunity to discuss the manuscript, and I thank you, Dr Goldstone, for a copy well in advance of the meeting. I have no disclosures.

I applaud the University of Pennsylvania group in helping us to understand the fate of mitral valve repair for various pathologies over the past many years. Their series is large enough to provide a clue into the effect on the right side of the heart.

Of your series of more than 4000 patients, your included cohort was only $10 \%$ of the total group, and of these, only $43 \%$ had grade 1 to 3 tricuspid regurgitation (TR). This seems like a small number according to the total group of 4000 patients. Was your exclusion criteria too aggressive?

Dr Goldstone. That is an excellent question. The 4200 patients were all-comers for mitral valve surgery, including any other concomitant procedure, such as a root replacement. One thousand patients had isolated mitral valve surgery, but that did include concomitant atrial ablation.

Because of the potential influence of atrial fibrillation on TR progression, and because recent studies have shown differences in etiology of mitral disease affecting progression of TR, we limited our study population to patients with degenerative mitral valve disease who underwent isolated mitral valve surgery, excluding patients with concomitant atrial ablations. That is why we ended up with 500 patients.

This was the particular patient cohort I wanted to look at because the most recent studies in patients with degenerative mitral valve disease argued against concomitant tricuspid annuloplasty, but our guidelines do not distinguish between disease etiology, and I think that is something that we need more data on moving forward.

Dr Zehr. The median follow-up time was 3.4 years. So this really is an intermediate-term follow-up study. Although you reported 10-year freedom from TR at 63\%, there were only 7 patients at risk at that time point. So if you back up and look at the 5-year follow-up, the freedom from significant TR was $90 \%$, which I would determine to be an excellent result.

So if you look at the data in a different way at an intermediate follow-up, the TR increase was relatively inconsequential with regard to symptoms and by grade. There is really only a $1.5+$ overall increase in patients with grade 0 to $2+$ preop, and indeed, among the 37 patients that had +3 preop, they actually got better over time at intermediate follow-up. No additional valve work is without risk, and tricuspid annuloplasty carries a small risk of increased need for pacemaker and an increased risk of endocarditis.
Should the conclusion be the opposite? That is, in patients with degenerative disease, the tricuspid valve should be left alone? Is this TR clinically relevant?

Dr Goldstone. That is a very good point and I think our conclusions are slightly controversial, but this is a controversial topic.

In terms of the midterm study, I agree, our median follow-up was 3.5 years, but that is why we conducted actuarial analyses, which demonstrate the overall trend. At the longest follow-up, more than 10 years after surgery, freedom from moderate or severe TR would be estimated to be $64 \%$. But the error bars do increase, so at 10 years there is a standard deviation of about $14 \%$.

Our data are fairly similar and go along with the other large studies in patients with degenerative mitral valve disease, such as that by Yilmaz and colleagues as well as the Cleveland Clinic study. Although the Mayo Clinic argued that nonsevere functional TR is unlikely to progress in these patients, they did use the mean grade of TR. Taking the mean of an ordinal variable can be misleading, especially because such data may be skewed. The authors did include a table that showed that at 5 years after surgery, $30 \%$ of patients had grade 3 or $4 \mathrm{TR}$, which is similar to the trend in our population. I believe the Cleveland Clinic data showed a similar trend, and actually at 3 years after surgery the patients with more significant preoperative TR who did not have a tricuspid valve repair already recurred to their prior grade of TR. In terms of the patients with moderate preoperative TR, they also seem to progress more quickly to recurrent moderate TR.

Dr Zehr. Seventy-three percent were reported to have follow-up echos. Do we know what happened to the other $27 \%$ of patients? Were they happy and healthy and thus no need for an echo or were they lost to follow-up?

Dr Goldstone. They were lost to follow-up.

Dr Steven F. Bolling (Ann Arbor, Mich). This is a very nice series in a controversial area. In light of the Dreyfus paper that shows that the addition of a tricuspid annuloplasty at the time of a mitral does not add to the operative mortality and in light of the Calafiore paper that says that the operative mortality may actually go down with the addition of a tricuspid annuloplasty in those patients, we applaud your aggressive approach.

In the real world, do you do a preventative tricuspid annuloplasty? What is your criteria now to do an annuloplasty while you are there?

Dr Goldstone. We just came across these data at this meeting. The reason we do not have a comparison group is because currently at our institution we will repair the tricuspid valve if there is concomitant severe TR. So our comparison group would have been only about 30 patients and they would have all had severe preoperative TR.

Dr Woo is going to become more aggressive with performing concomitant tricuspid annuloplasty in patients with a dilated tricuspid annulus who are undergoing mitral valve surgery.

Dr Bolling. In light of those data, do you think this calls for a randomized, large prospective trial in this group of patients?

Dr Goldstone. I have been trying to come up with a good way to conduct a randomized controlled trial in this group of patients for quite some time. The main issue that we run into is selecting a satisfiable clinical end point. First of all, the trial would need to be fairly long depending on what type of mitral valve disease etiology is selected in the study. Progression of TR 
is definitely faster in patients with functional or ischemic mitral regurgitation, and I think that has to do with concomitant right ventricular dysfunction in those patients versus a patient with mitral valve prolapse. But you are still going to have to create a study that spans many years, as well as choose an appropriate clinical end point. Unfortunately, in my opinion New York Heart Association functional class is a poor metric for right heart function. I did not have data on diuretic use, liver transaminases, or kidney function. I believe that in a clinical trial a surrogate end point, such as right ventricular dimensions or function in addition to grade of tricuspid regurgitation, would need to be used.

However I definitely agree that a randomized prospective trial would be ideal to help inform the cardiac surgery community on this controversial issue.

Dr Harold L. Lazar (Boston, Mass). Did you measure pulmonary artery (PA) pressures either by echo or by cath and have you found that to be a determinant of what ultimately happens to the TR? I have seen that patients who have minimal TR but have high PA pressures at the time of mitral valve replacement or repair tend to be the ones who tend to have increased TR in the future.

Dr Goldstone. We did look at PA pressures. They were measured by right heart catheterization, and preoperative mean PA pressure was not a significant predictor of progression of TR postoperatively in univariate analysis. That is consistent with the Mayo paper, which did not find pulmonary artery pressure to be a significant predictor either. I agree that it makes sense that pulmonary hypertension would influence TR progression. Perhaps the reason why different studies identify different predictors of TR progression alludes to the multifactorial nature of functional TR.

Dr Lazar. In those patients who had progressive TR, did their PA pressures go up or did they stay the same?

Dr Goldstone. I do not have longitudinal data on PA pressure.

\title{
EDITORIAL COMMENTARY
}

\section{The role of cognitive dissonance in the management of functional tricuspid regurgitation at the time of degenerative mitral valve repair}

\author{
Rakesh M. Suri, MD, DPhil, ${ }^{\mathrm{a}}$ and Yan Topilsky, $\mathrm{MD}^{\mathrm{b}}$
}

See related article on pages 2802-10.

Cognitive dissonance: Psychologic conflict resulting from incongruous beliefs and attitudes held simultaneously.

In this issue of the Journal, Goldstone and colleagues ${ }^{1}$ from the University of Pennsylvania and Stanford have assembled the third largest series $(n=495)$ in the literature of patients with mitral valve prolapse undergoing mitral valve repair with coexistent functional tricuspid valve regurgitation (TR) not surgically managed. Forty-three percent of patients had anywhere between trivial to moderately severe TR at mitral valve repair, and freedom

From the Division of Cardiovascular Surgery, ${ }^{\mathrm{a}}$ Mayo Clinic, Rochester, Minn; and the Tel Aviv Sourasky Medical Center, ${ }^{\mathrm{b}}$ Tel Aviv, Israel.

Disclosures: Authors have nothing to disclose with regard to commercial support.

Received for publication Aug 25, 2014; accepted for publication Aug 25, 2014; available ahead of print Oct 3, 2014

Address for reprints: Rakesh M. Suri, MD, DPhil, Division of Cardiovascular Surgery, Mayo Clinic College of Medicine, 200 First St SW, Rochester, MN 55905 (E-mail: suri.rakesh@mayo.edu).

J Thorac Cardiovasc Surg 2014;148:2810-2

$0022-5223 / \$ 36.00$

Copyright (c) 2014 by The American Association for Thoracic Surgery

http://dx.doi.org/10.1016/j.jtcvs.2014.08.040 from greater than moderate TR was approximately $60 \%$ at 9 years. Older age and grade of preoperative TR predicted progression; and "some degree" of right ventricular dysfunction was more frequent in those with severe TR during follow-up.

What does this study tell us? The findings reaffirm those published from the Mayo Clinic in 2011 and in 2014. ${ }^{2,3}$ Our institution's first series studied 699 patients who underwent mitral valve repair for isolated degenerative leaflet prolapse and moderate or less coexistent functional TR. We identified that moderate TR actually regressed with time in most cases after successful and durable repair of degenerative mitral valve disease. Indeed, only 1 patient out of the entire series required reoperation for progressive TR 4.5 years after mitral valve repair. Our most recent report expanded on this analysis, studying 747 adult patients with mitral valve prolapse among whom 683 underwent mitral valve repair and 64 had replacement. We found no difference in the occurrence of moderately severe or severe TR after mitral valve repair versus replacement and found the univariate predictors of TR progression to be older age, female sex, higher pulmonary arterial pressure, and larger left atrial size. Only 2 patients required reoperation for late functional TR. The report of Goldstone and colleagues ${ }^{1}$ thus adds to 